\title{
Comparação Entre Diferentes Formulações do Problema de Fluxo de Potência Ótimo Utilizando o Método de Pontos Interiores
}

\author{
A.R.L. OLIVEIRA ${ }^{1}$, A.M. LIMA ${ }^{2}$ IMECC - Instituto de Matemática, Estatística, e \\ Ciência da Computação, UNICAMP, Praça Sérgio Buarque de Holanda 651, 13081- \\ 970 Campinas, SP, Brasil.
}

\begin{abstract}
Resumo. Neste trabalho, os problemas de fluxo de potência ótimo DC utilizando o modelo de fluxo em redes e o princípio do mínimo esforço são apresentados. Em seguida, um método de pontos interiores preditor-corretor específico é desenvolvido para os dois modelos de fluxo de potência. Resultados numéricos em MATLAB comparando as duas abordagens para sistemas reais de grande porte são apresentados e o método de pontos interiores se mostra bastante robusto, convergindo rapidamente para todos os casos testados.
\end{abstract}

\section{Introdução}

O objetivo deste trabalho consiste em comparar duas abordagens do problema de fluxo de potência ótimo utilizando o método de pontos interiores preditor-corretor $[6,4]$, que é o método de pontos interiores mais utilizado na prática.

O problema de fluxo de potência linearizado DC é representado por um modelo de fluxos em redes com restrições adicionais [1]. Aplicamos o princípio do mínimo esforço [7] a este problema obtendo, assim, uma outra formulação do fluxo de potência ótimo. Desenvolvemos, então, o método de pontos interiores preditorcorretor $[6,4,12]$ para as duas formulações mencionadas. Em [8], os métodos de pontos interiores primais-duais são desenvolvidos e seu desempenho é comparado para ambas formulações.

O problema de fluxo de potência ótimo DC é de grande importância na área de sistemas de potência, servindo como base para diversas outras aplicações. O surgimento dos métodos de pontos interiores trouxe à tona uma nova linha de pesquisa nesta área. Estes métodos são reconhecidos por sua robustez [11, 5], principalmente devido ao tratamento eficiente de desigualdades.

\footnotetext{
19aurelio@ime.unicamp.br

2 amlima@ime.unicamp.br.
} 


\section{Problema de Fluxo de Potência Ótimo DC e o Princípio do mínimo Esforço}

O problema quadrático de fluxo de potência ótimo (FPO) DC pode ser representado por um problema de fluxo em redes com restrições adicionais [10], como segue:

$$
\begin{array}{ll}
\min & \alpha f^{t} R f+\beta\left(p^{t} H p+c^{t} p\right) \\
\text { s.a. } & A f=p-l, T f=0 \\
& f^{\text {min }} \leq f \leq f^{\text {max }}, p^{\text {min }} \leq p \leq p^{\text {max }}
\end{array}
$$

onde:

$f$ representa o vetor de fluxo de potência ativa;

$p$ representa o vetor de geração de potência ativa;

$R$ representa a matriz diagonal das resistências das linhas;

$H$ representa a componente quadrática do custo de geração;

$c$ representa a componente linear do custo de geração;

$A$ representa a matriz de incidência da rede de transmissão;

$T$ representa a matriz de reatância da rede de transmissão;

$l$ representa o vetor demanda de potência ativa;

$f^{\max }, f^{\min }, p^{\max }$ e $p^{\text {min }}$ são os vetores de limites de fluxo e de geração de potência ativa;

$\alpha$ e $\beta$ são ponderações dos objetivos a minimizar.

Para simplificar o desenvolvimento do método, faremos as seguintes alterações no modelo:

- Mudança de variáveis $\tilde{f}=f-f^{\text {min }}$ e $\tilde{p}=p-p^{\text {min }}$, reduzindo o número de variáveis de folga do problema.

- As ponderações $\alpha$ e $\beta$ serão incorporadas às matrizes $R$ e $H$, respectivamente, e $\beta$ ao vetor $c$.

Após estas alterações, as condições de otimalidade são dadas por:

factibilidade primal: $\left\{\begin{array}{rl}A f-p & =l_{i} \\ T f & =l_{v} \\ f+s_{f} & =f^{\text {max }} \\ p+s_{p} & =p^{\text {max }} \\ \left(f, s_{f}\right) & \geq 0 \\ \left(p, s_{p}\right) & \geq 0\end{array}\right.$,

factibilidade dual: $\left\{\begin{aligned} C^{t} y+z_{f}-w_{f}-R f & =c_{f} \\ -y(p)+z_{p}-w_{p}-H p & =c_{p} \\ \left(z_{p}, w_{p}\right) & \geq 0 \\ \left(z_{f}, w_{f}\right) & \geq 0\end{aligned}\right.$ 
condições de complementaridade: $\left\{\begin{aligned} F Z_{f} e & =0 \\ P Z_{p} e & =0 \\ S_{f} W_{f} e & =0 \\ S_{p} W_{p} e & =0\end{aligned}\right.$,

onde $c_{f}=2 R f^{\text {min }}, c_{p}=2 H p^{\text {min }}+c, l_{i}=p^{\text {min }}-l-A f^{\text {min }}, l_{v}=-T f^{\text {min }}$, $s_{f}=f^{\max }-f$ e $s_{p}=p^{\max }-p$ são as variáveis de folga do problema primal, $z_{f} \mathrm{e}$ $z_{p}$ são as variáveis de folga do problema dual, $C^{t}=\left(\begin{array}{ll}A^{t} & T^{t}\end{array}\right)$ e $y(p)$ representa os elementos da variável dual $y$ correspondentes às barras de geração. Utilizamos a notação $F=\operatorname{diag}(f)$ e $e=(1,1, \ldots, 1)^{t}$.

O princípio do mínimo esforço consiste na simplificação do modelo de fluxo de potência ativa linearizado (2.1), correspondente a um fluxo em redes, antes da aplicação de um método de otimização.

Aplicando ao problema (2.1) o princípio do mínimo esforço (PME), com as simplificações definidas anteriormente, ou seja, substituindo a relação $f=X^{-1} A^{t} \theta$, onde:

$X$ representa a matriz diagonal das reatâncias das linhas;

$\theta$ representa o vetor dos ângulos das tensões;

e ignorando a restrição $T f=0$, obtemos:

$$
\begin{array}{ll}
\min & \theta^{t} \tilde{B} \theta+p^{t} H p+c^{t} p \\
\text { s.a. } & B \theta-p=-l \\
& 0 \leq p \leq p^{\max }
\end{array}
$$

onde $B=A X^{-1} A^{t}$ e $\tilde{B}=A X^{-t} R X^{-1} A^{t}$.

Para o qual as condições de otimalidade são:

factibilidade primal: $\left\{\begin{array}{rl}B \theta-p & =l \\ p+s_{p} & =p^{\max } \\ \left(p, s_{p}\right) & \geq 0\end{array}\right.$,

factibilidade dual: $\left\{\begin{aligned} B^{t} y-\tilde{B} \theta & =0 \\ -y(p)+z_{p}-w_{p}-H p & =c_{p} \\ \left(z_{p}, w_{p}\right) & \geq 0\end{aligned}\right.$,

condições de complementaridade: $\left\{\begin{array}{r}P Z_{p} e=0 \\ S_{p} W_{p} e=0\end{array}\right.$.

Então, de posse das condições de otimalidade, desenvolveremos o método de pontos interiores preditor-corretor para estas duas abordagens do problema de fluxo de potência ótimo (FPO e PME). Na segunda abordagem, um conjunto de variáveis $\theta$ substitui as variáveis $f$ do problema original, reduzindo a dimensão do problema. No entanto, não é possível afirmar de antemão que esta redução leva a um método mais eficiente, uma vez que as iterações dos métodos desenvolvidos para ambos modelos geram direções distintas [3]. 


\section{Método Preditor-Corretor Aplicado ao Modelo de Fluxos em Rede}

A idéia do método preditor-corretor desenvolvido em $[6,4]$ consiste em utilizar uma direção que contenha três componentes:

- Direção Afim-Escala (direção preditora ou de Newton).

- Direção de Centragem.

- Direção de Correção, que busca compensar a aproximação linear do método de Newton.

Ou seja, primeiramente é calculada a direção afim. A segunda direção é calculada utilizando o mesmo Jacobiano, para que o esforço computacional por iteração não duplique. Então, temos de resolver dois sistemas lineares para determinar as direções.

Primeiramente, é calculada a direção afim $(d \tilde{x}, d \tilde{y}, d \tilde{t})$, onde $d \tilde{x}=\left(d \tilde{f}, d \tilde{p}, d \tilde{s_{f}}, d \tilde{s_{p}}\right)$ e $d \tilde{t}=\left(d \tilde{z_{f}}, d \tilde{w}_{f}, d \tilde{z_{p}}, d \tilde{w}_{p}\right)$, resolvendo o sistema linear abaixo:

$$
\left\{\begin{aligned}
A d \tilde{f}-d \tilde{p} & =r_{i} \\
T d \tilde{f} & =r_{v} \\
d \tilde{f}+d \tilde{s_{f}} & =r_{f} \\
d \tilde{p}+d \tilde{s_{p}} & =r_{p} \\
C^{t} d \tilde{y}+d \tilde{z_{f}}-d \tilde{w_{f}}-R d \tilde{f} & =r_{y} \\
-d y(p)+d \tilde{z_{p}}-d \tilde{w_{p}}-H d \tilde{p} & =r_{g} \\
Z_{f} d \tilde{f}+F d \tilde{z_{f}} & =r_{z f} \\
Z_{p} d \tilde{p}+P d \tilde{z_{p}} & =r_{z p} \\
W_{f} d \tilde{s_{f}}+S_{f} d \tilde{w_{f}} & =r_{w f}^{\tilde{w} f} \\
W_{p} d \tilde{s_{p}}+S_{p} d \tilde{w_{p}} & =r_{w p}^{\tilde{w}}
\end{aligned}\right.
$$

onde os resíduos são

$$
\left\{\begin{array}{rl}
r_{i} & =l_{i}+p-A f \\
r_{v} & =l_{v}-T f \\
r_{f} & =f^{\max }-f-s_{f} \\
r_{p} & =p^{\max }-p-s_{p} \\
r_{y} & =c_{f}-C^{t} y-z_{f}+w_{f}+R f \\
r_{g} & =c_{p}+y(p)-z_{p}+w_{p}+H p \\
r_{z f}^{\tilde{v}} & =-F Z_{f} e \\
r_{z p}^{\sim} & =-P Z_{p} e \\
r_{w f}^{\tilde{w} f} & =-S_{f} W_{f} e \\
r_{w p}^{\tilde{w}} & =-S_{p} W_{p} e
\end{array} .\right.
$$

Em seguida, a direção desejada é obtida substituindo as quatro últimas equações de (3.1) por:

$$
\left\{\begin{aligned}
Z_{f} d f+F d z_{f} & =r_{z f} \\
Z_{p} d p+P d z_{p} & =r_{z p} \\
W_{f} d s_{f}+S_{f} d w_{f} & =r_{w f} \\
W_{p} d s_{p}+S_{p} d w_{p} & =r_{w p}
\end{aligned}\right.
$$


e resolvendo-se o sistema linear resultante, onde:

$$
\left\{\begin{array}{rl}
r_{z f} & =\mu e-F Z_{f} e-\Delta \tilde{F} \Delta \tilde{Z}_{f} \\
r_{z p} & =\mu e-P Z_{p} e-\Delta \tilde{P} \Delta \tilde{Z}_{p} \\
r_{w f} & =\mu e-S_{f} W_{f} e-\Delta \tilde{S}_{f} \Delta \tilde{W}_{f} \\
r_{w p} & =\mu e-S_{p} W_{p} e-\Delta \tilde{S}_{p} \Delta \tilde{W}_{p}
\end{array} .\right.
$$

O cálculo de $\mu$ é função da direção afim. Quanto melhor a direção afim, menor a perturbação e vice-versa. Ou seja:

$\gamma=x^{t} t, \tilde{\gamma}=\left(x+\tilde{\alpha_{p}} d \tilde{x}\right)^{t}\left(t+\tilde{\alpha_{d}} d \tilde{t}\right)$ e $\mu=\sigma\left(\frac{\gamma}{n_{p}}\right)$, onde

$$
\sigma=\left\{\begin{array}{c}
\left(\frac{\tilde{\gamma}}{\gamma}\right)^{3}, \text { se } \gamma \geq 1 \\
\left(\frac{\tilde{\gamma}}{\sqrt{n_{p}}}\right), \text { se } \gamma<1
\end{array}\right.
$$

Os sistemas (3.1) e (3.2) podem ter suas dimensões substancialmente reduzidas através de eliminação de variáveis

$$
\left(C D_{f}^{-1} C^{t}+D\right) d y=r,
$$

onde $r=\tilde{r}+C D_{f}^{-1} r_{a}-D r_{b}$.

O fato de a matriz $C$ ter dimensão $(n+1) \times n$ é bastante relevante. Esta característica pode ser utilizada para reduzir o esforço computacional por iteração dos métodos de pontos interiores, assim, se formarmos a matriz não singular $\tilde{C}=$ $\left[\begin{array}{ll}C & e_{j}\end{array}\right]$, onde $e_{j}$ representa um vetor canônico (vetor formado pelas colunas da matriz identidade), onde $j$ é um índice referente a uma barra de geração, o sistema (3.3) pode ser reescrito na seguinte forma:

$$
\left(\tilde{C} \tilde{D}_{f}^{-1} \tilde{C}^{t}+\tilde{D}\right) d y=r
$$

onde $\tilde{D}_{f}^{-1}$ incorpora o elemento diagonal retirado de $D$ para formar $\tilde{D}$. A resolução de (3.4) se dá em duas etapas. Na primeira, um sistema linear contendo apenas a matriz $\tilde{C} \tilde{D}_{f}^{-1} \tilde{C}^{t}$ é resolvido. Vale observar que como $\tilde{C}$ é quadrada, a resolução do sistema com a matriz $\tilde{C} \tilde{D}_{f}^{-1} \tilde{C}^{t}$ fica muito barata, pois apenas a matriz diagonal $\tilde{D}_{f}$ varia a cada iteração. Então, apenas uma decomposição de $\tilde{C}$ é necessária e pode ser realizada antes da aplicação do método iterativo.

Na segunda etapa, a fórmula de Sherman-Morrison-Woodbury [2] é aplicada para a obtenção de $d y$

$$
\begin{aligned}
W & =\tilde{C}^{-1} E \\
Z & =W^{t} \tilde{D}_{f} W \\
v & =\left(\tilde{C} \tilde{D}_{f}^{-1} \tilde{C}^{t}\right)^{-1} r \\
d y & =v-\left(\tilde{C} \tilde{D}_{f}^{-1} \tilde{C}^{t}\right)^{-1} E\left(\tilde{D}^{-1}+Z\right)^{-1} E^{t} v,
\end{aligned}
$$

onde $E$ é formada por vetores canônicos correspondentes aos elementos diagonais não nulos de $\tilde{D}$. Consequentemente, $W$ é fixa para uma determinada rede e pode ser calculada antes da aplicação do método iterativo. 
Obtidas as direções, o método preditor-corretor pode ser resumido como segue: Dados $\left(f^{0}, p^{0}, s_{f}^{0}, s_{p}^{0}, z_{f}^{0}, w_{f}^{0}, z_{p}^{0}, w_{p}^{0}\right) \geq 0, y^{0}$ livre e $\tau \in(0,1)$.

Sejam $x=\left(f, p, s_{f}, s_{p}\right), t=\left(z_{f}, w_{f}, z_{p}, w_{p}\right), n_{p}=\operatorname{dim}(x)$ e $\tilde{C}=\left[\begin{array}{ll}C & e_{j}\end{array}\right]$.

Calcule $W=\tilde{C}^{-1} E$.

Para $k=0,1,2, \ldots$, faça:

Calcule os resíduos $r_{i}^{k}, r_{v}^{k}, r_{f}^{k}, r_{p}^{k}, r_{y}^{k}, r_{g}^{k}, r_{z f}{ }^{k}, r_{z p}{ }^{k}, r_{w f}{ }^{k}$ e $r_{w p}^{{ }^{k}}$.

$$
\begin{aligned}
& r_{a}^{k}=r_{y}^{k}-\left(F^{k}\right)^{-1} r_{z f}^{\tilde{k}}+\left(S_{f}^{k}\right)^{-1} r_{w f}^{\tilde{w}}-\left(S_{f}^{k}\right)^{-1} W_{f}^{k} r_{f}^{k} \\
& r_{b}^{k}=r_{g}^{k}-\left(P^{k}\right)^{-1} r_{z p}{ }^{k}+\left(S_{p}^{k}\right)^{-1} r_{w p}{ }^{k}-\left(S_{p}^{k}\right)^{-1} W_{p}^{k} r_{p}^{k} \\
& D_{f}^{k}=\left(F^{k}\right)^{-1} Z_{f}^{k}+\left(S_{f}^{k}\right)^{-1} W_{f}^{k}+R \\
& r^{k}=\left(\begin{array}{c}
r_{i}{ }^{k} \\
r_{v}{ }^{k}
\end{array}\right)+C\left(D_{f}^{k}\right)^{-1} r_{a}^{k}-D r_{b}^{k} \\
& Z^{k}=W^{t} \tilde{D}_{f}^{k} W \\
& v^{k}=\left(\tilde{C}^{-t}\left(\tilde{D}_{f}^{k}\right)^{-1} \tilde{C}^{t}\right)^{-1} r^{k} \\
& d \tilde{y}^{k}=v^{k}-\left(\tilde{C}^{-t}\left(\tilde{D}_{f}^{k}\right)^{-1} \tilde{C}^{t}\right)^{-1} E\left(\tilde{D}^{-1}+Z^{k}\right)^{-1} E^{t} v^{k} \\
& d \tilde{f}^{k}=-\left(D_{f}^{k}\right)^{-1}\left(r_{a}^{k}-C^{t} d \tilde{y}^{k}\right) \\
& d y \tilde{(p)})^{k}=\left(\begin{array}{c}
-\left(D_{p}^{k}\right)^{-1} \\
0
\end{array}\right) d \tilde{y}^{k} \\
& D_{p}^{k}=\left(P^{k}\right)^{-1} Z_{p}^{k}+\left(S_{p}^{k}\right)^{-1} W_{p}^{k}+H \\
& d \tilde{p}^{k}=-\left(D_{p}^{k}\right)^{-1}\left(r_{b}^{k}+d y \tilde{y}(p)^{k}\right) \\
& d{\tilde{s_{f}}}^{k}=r_{f}^{k}-d \tilde{f}^{k} \\
& d \tilde{s}^{k}=r_{2}^{k}-d \tilde{p}^{k} \\
& d \tilde{z_{f}}{ }^{k}=\left(Z^{k}\right)^{-1}\left(\tilde{r}_{z f}^{k}-Z_{f}^{k} d \tilde{f}^{k}\right) \\
& d \tilde{z_{p}}{ }^{k}=\left(P^{k}\right)^{-1}\left(\tilde{r}_{z p}{ }^{k}-Z_{p}^{k} d \tilde{p}^{k}\right) \\
& d \tilde{w}_{f}^{k}=\left(S_{f}^{k}\right)^{-1}\left(\tilde{r}_{w f}^{k}-W_{f}^{k} d{\tilde{s_{f}}}^{k}\right) \\
& d \tilde{w}_{p}{ }^{k}=\left(S_{p}^{k}\right)^{-1}\left(\tilde{r}_{w p}{ }^{k}-W_{p}^{k} d \tilde{s_{p}}{ }^{k}\right) \\
& d \tilde{x}^{k}=\left(d \tilde{f}^{k}, d \tilde{p}^{k}, d{\tilde{s_{f}}}^{k}, d{\tilde{s_{p}}}^{k}\right) \\
& d \tilde{t}^{k}=\left(d \tilde{z_{f}}{ }^{k}, d \tilde{w}_{f}^{k}, d{\tilde{z_{p}}}^{k}, d \tilde{w}_{p}{ }^{k}\right) \\
& {\tilde{\rho_{p}}}^{k}=\min _{d \tilde{x}^{k}<0}\left\{-\frac{x_{i}{ }^{k}}{d \tilde{x}_{i}{ }^{k}}\right\} ; \quad{\tilde{\rho_{d}}}^{k}=\min _{d \tilde{t}_{i}{ }^{k}<0}\left\{-\frac{t_{i}{ }^{k}}{d \tilde{t}_{i}{ }^{k}}\right\} \\
& {\tilde{\alpha_{p}}}^{k}=\min \left(1, \tau{\tilde{\rho_{p}}}^{k}\right) ; \quad{\tilde{\alpha_{d}}}^{k}=\min \left(1, \tau{\tilde{\rho_{d}}}^{k}\right) \\
& \tilde{\gamma}^{k}=\left(x^{k}+{\tilde{\alpha_{p}}}^{k} d \tilde{x}^{k}\right)^{t}\left(t^{k}+{\tilde{\alpha_{d}}}^{k} d \tilde{t}^{k}\right)
\end{aligned}
$$

Calcule $\sigma^{k}$ e $\mu^{k}$.

Calcule os resíduos $r_{z f}^{k}, r_{z p}^{k}, r_{w f}^{k}$ e $r_{w p}^{k}$.

$$
r_{s a}^{k}=r_{y}^{k}-\left(F^{k}\right)^{-1} r_{z f}^{k}+\left(S_{f}^{k}\right)^{-1} r_{w f}^{k}-\left(S_{f}^{k}\right)^{-1} W_{f}^{k} r_{f}^{k}
$$




$$
\begin{aligned}
r_{s b}^{k} & =r_{g}^{k}-\left(P^{k}\right)^{-1} r_{z p}^{k}+\left(S_{p}^{k}\right)^{-1} r_{w p}^{k}-\left(S_{p}^{k}\right)^{-1} W_{p}^{k} r_{p}^{k} \\
r^{k} & =\left(\begin{array}{c}
r_{i}{ }^{k} \\
{ }^{k}
\end{array}\right)+C\left(D_{f}^{k}\right)^{-1} r_{s a}^{k}-D r_{b}^{k} \\
v^{k} & =\left(\tilde{C}^{-t}\left(\tilde{D}_{f}^{k}\right)^{-1} \tilde{C}^{t}\right)^{-1} r^{k} \\
d y^{k} & =v^{k}-\left(\tilde{C}^{-t}\left(\tilde{D}_{f}^{k}\right)^{-1} \tilde{C}^{t}\right)^{-1} E\left(\tilde{D}^{-1}+Z^{k}\right)^{-1} E^{t} v^{k} \\
d f^{k} & =-\left(D_{f}^{k}\right)^{-1}\left(r_{s a}^{k}-C^{t} d y^{k}\right) \\
d y(p)^{k} & =\left(\begin{array}{c}
-\left(D_{p}^{k}\right)^{-1} \\
0
\end{array}\right) d y^{k} \\
D_{p}^{k} & =\left(P^{k}\right)^{-1} Z_{p}^{k}+\left(S_{p}^{k}\right)^{-1} W_{p}^{k}+H \\
d p^{k} & =-\left(D_{p}^{k}\right)^{-1}\left(r_{s b}^{k}+d y(p)^{k}\right) \\
d s_{f}^{k} & =r_{f}^{k}-d f^{k} \\
d s_{p}^{k} & =r_{2}^{k}-d p^{k} \\
d z_{f}^{k} & =\left(Z^{k}\right)^{-1}\left(r z f^{k}-Z_{f}^{k} d f^{k}\right) \\
d z_{p}^{k} & =\left(P^{k}\right)^{-1}\left(r_{z p}^{k}-Z_{p}^{k} d p^{k}\right) \\
d w_{f}^{k} & =\left(S_{f}^{k}\right)^{-1}\left(r_{w f}^{k}-W_{f}^{k} d s_{f}^{k}\right) \\
d w_{p}^{k} & =\left(S_{p}^{k}\right)^{-1}\left(r_{w p}^{k}-W_{p}^{k} d s_{p}^{k}\right) \\
d x^{k} & =\left(d f^{k}, d p^{k}, d s f^{k}, d s_{p}^{k}\right) \\
d t^{k} & =\left(d z_{f}^{k}, d w_{f}^{k}, d z_{p}^{k}, d w_{p}^{k}\right) \\
\rho_{p}{ }^{k} & =\min _{d x^{k}<0}\left\{-\frac{x_{i}{ }^{k}}{d x_{i}{ }^{k}}\right\} ; \quad \rho_{d}^{k}=\min _{d t_{i}{ }^{k}<0}\left\{\frac{t_{i}{ }^{k}}{d t_{i}^{k}}\right\} \\
\alpha_{p}^{k} & =m i n\left(1, \tau \rho_{p}^{k}\right) ; \quad \alpha_{d}^{k}=\min ^{k}\left(1, \tau \rho_{d}^{k}\right) \\
\tilde{\gamma}^{k} & =\left(x^{k}+\alpha_{p}^{k} d x^{k}\right)^{t}\left(t^{k}+\alpha_{d}^{k} d t^{k}\right) \\
x^{k+1} & =x^{k}+\alpha_{p}^{k} d x^{k} \\
y^{k+1} & =y^{k}+\alpha_{d}^{k} d y^{k} \\
t^{k+1} & =t^{k}+\alpha_{d}^{k} d t^{k} \\
&
\end{aligned}
$$

Até convergir.

O maior esforço do método consiste na resolução do sistema linear resultante da eliminação de variáveis. Para resolver este sistema utilizamos a fórmula de ShermanMorrison-Woodbury [2], o que nos permite reduzir bastante o esforço computacional [9]. A matriz $\tilde{C}$ é uma matriz quadrada, que só depende dos dados do problema e, então apenas uma decomposição dessa matriz é necessária e pode ser realizada antes do processo iterativo.

\subsection{Inicialização, Parâmetros e Critério de Convergência}

Para a implementação do método de pontos interiores utilizaremos:

$$
\text { - } \tau=0.99995 ; \sigma=\frac{1}{\sqrt{n}} \text {. }
$$


O ponto inicial adotado foi:

- $f^{0}=s_{f}^{0}=\frac{p^{\max }}{2} ; p^{0}=s_{p}{ }^{0}=\frac{p^{\max }}{2} ; y^{0}=0$;

- $z_{f}{ }^{0}=w_{f}^{0}=(R+I) e ; z_{p}{ }^{0}=w_{p}^{0}=e$.

O critério de convergência é dado por:

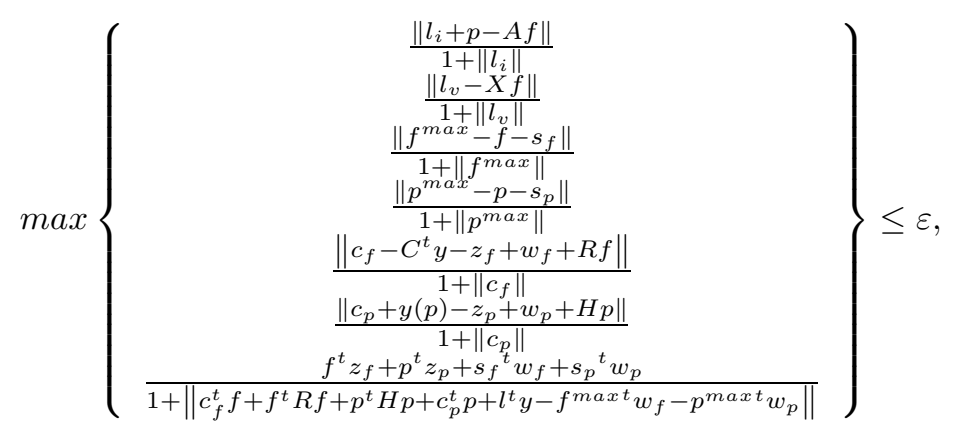

onde $\varepsilon$ é a tolerância estabelecida.

\section{Método Preditor-Corretor Aplicado à Formulação Usando o Princípio do Mínimo Esforço}

De forma similar ao modelo anterior, calculamos a direção afim, resolvendo o sistema linear abaixo

$$
\left\{\begin{aligned}
B d \tilde{\theta}-d \tilde{p} & =r_{1} \\
d \tilde{p}+d \tilde{s_{p}} & =r_{2} \\
B^{t} d \tilde{y}-\tilde{B} d \tilde{\theta} & =r_{3} \\
-d y(p)+d \tilde{z_{p}}-d \tilde{w_{p}}-H d \tilde{p} & =r_{4} \\
Z_{p} d \tilde{p}+P d \tilde{z_{p}} & =\tilde{r_{5}} \\
W_{p} d \tilde{s_{p}}+S_{p} d \tilde{w_{p}} & =\tilde{r_{6}}
\end{aligned}\right.
$$

Onde os resíduos são dados por:

$$
\left\{\begin{array}{rl}
r_{1} & =l-B \theta+p \\
r_{2} & =p^{\max }-p-s_{p} \\
r_{3} & =-B^{t} y+\tilde{B} \theta \\
r_{4} & =c_{p}+y(p)-z_{p}+w_{p}+H p \\
\tilde{r_{5}} & =-P Z_{p} e \\
\tilde{r_{6}} & =-S_{p} W_{p} e
\end{array} .\right.
$$

Em seguida, a direção desejada é obtida substituindo as duas últimas equações de (4.1) por

$$
\left\{\begin{aligned}
Z_{p} d p+P d z_{p} & =r_{5} \\
W_{p} d s_{p}+S_{p} d w_{p} & =r_{6}
\end{aligned}\right.
$$


e resolvendo-se o sistema linear resultante, onde

$$
\left\{\begin{array}{l}
r_{5}=\mu e-P Z_{p} e-\Delta \tilde{P} \Delta \tilde{Z}_{p} \\
r_{6}=\mu e-S_{p} W_{p} e-\Delta \tilde{S}_{p} \Delta \tilde{W}_{p}
\end{array} .\right.
$$

Podemos reduzir os sistemas acima, através da eliminação de variáveis, a

$$
B\left(\tilde{B}^{-1}\left(B^{t} d y-r_{3}\right)\right)+D d y=r_{a} \Longrightarrow\left(B \tilde{B}^{-1} B^{t}+D\right) d y=r,
$$

onde $r=r_{a}+B \tilde{B}^{-1} r_{3}$.

Utilizaremos novamente a fórmula de Sherman-Morrison-Woodbury [2] para obter $d y$

$$
\begin{aligned}
W & =B^{-1} E \\
Z & =W^{t} \tilde{B} W \\
v & =\left(B \tilde{B}^{-1} B^{t}\right)^{-1} r=\left(B^{-t} \tilde{B} B^{-1}\right) r \\
d y & =v-\left(B \tilde{B}^{-1} B^{t}\right)^{-1} E\left(D^{-1}+Z\right)^{-1} E^{t} v,
\end{aligned}
$$

onde $E$ é formada por vetores canônicos correspondentes aos elementos diagonais não nulos de $D$. $W$ e $Z$ são fixas para uma determinada rede e podem ser calculadas antes da aplicação do método iterativo.

Assim, obtemos o método de pontos interiores preditor-corretor abaixo

Dados $\left(p^{0}, s_{p}^{0}, z_{p}^{0}, w_{p}^{0}\right) \geq 0, \theta^{0}$ e $y^{0}$ livres e $\tau \in(0,1)$.

Sejam $x=\left(p, s_{p}\right), t=\left(z_{p}, w_{p}\right)$ e $n_{p}$ a dimensão do vetor $x$.

Calcule $W=B^{-1} E$ e $Z=W^{t} \tilde{B} W$.

Para $k=0,1,2, \ldots$, faça:

Calcule os resíduos $r_{1}^{k}$ a $r_{4}^{k},{\tilde{r_{5}}}^{k}$ e ${\tilde{r_{6}}}^{k}$.

$$
\begin{aligned}
r_{p}^{k} & =r_{4}^{k}-\left(P^{k}\right)^{-1}{\tilde{r_{5}}}^{k}+\left(S_{p}^{k}\right)^{-1}{\tilde{r_{6}}}^{k}-\left(S_{p}^{k}\right)^{-1} W_{p}^{k} r_{2}^{k} \\
D_{p}^{k} & =\left(P^{k}\right)^{-1} Z_{p}^{k}+H+\left(S_{p}^{k}\right)^{-1} W_{p}^{k} \\
r_{a}^{k} & =r_{1}^{k}+D^{k} r_{p}^{k} \\
r^{k} & =r_{a}^{k}+B \tilde{B}^{-1} r_{3}^{k} \\
v^{k} & =\left(B^{-t} \tilde{B} B^{-1}\right) r^{k} \\
d \tilde{y}^{k} & =v^{k}-\left(B^{-t} \tilde{B} B^{-1}\right) E\left(D^{-1}+Z\right)^{-1} E^{t} v^{k} \\
d \tilde{\theta}^{k} & =\tilde{B}^{-1}\left(B^{t} d \tilde{y}^{k}-r_{3}^{k}\right) \\
d \tilde{p}^{k} & =-\left(D_{p}^{k}\right)^{-1}\left(r_{p}^{k}+d y(p)^{k}\right) \\
d \tilde{s_{p}}{ }^{k} & =r_{2}^{k}-d \tilde{p^{k}} \\
d{\tilde{z_{p}}}^{k} & =\left(P^{k}\right)^{-1}\left({\tilde{r_{5}}}^{k}-Z_{p}^{k} d \tilde{p}^{k}\right) \\
d{\tilde{w_{p}}}^{k} & =\left(S_{p}^{k}\right)^{-1}\left({\tilde{r_{6}}}^{k}-W_{p}^{k} d{\tilde{s_{p}}}^{k}\right) \\
d \tilde{x}^{k} & =\left(d \tilde{p}^{k}, d \tilde{s_{p}}{ }^{k}\right) \\
d \tilde{t}^{k} & =\left(d{\tilde{z_{p}}}^{k}, d{\tilde{w_{p}}}^{k}\right) \\
{\tilde{\rho_{p}}}^{k} & =\min _{d \tilde{x}_{i}^{k}<0}\left\{-\frac{x_{i}{ }^{k}}{d \tilde{x}_{i}{ }^{k}}\right\} ; \quad{\tilde{\rho_{d}}}^{k}=\min _{d \tilde{t}_{i}^{k}<0}\left\{-\frac{t_{i}{ }^{k}}{d \tilde{t}_{i}^{k}}\right\}
\end{aligned}
$$




$$
\begin{aligned}
{\tilde{\alpha_{p}}}^{k} & =\min \left(1, \tau{\tilde{\rho_{p}}}^{k}\right) ; \quad{\tilde{\alpha_{d}}}^{k}=\min \left(1, \tau{\tilde{\rho_{d}}}^{k}\right) ; \quad \alpha^{k}=\min \left(\alpha_{p}, \alpha_{d}\right) \\
\tilde{\gamma}^{k} & =\left(x^{k}+{\tilde{\alpha_{p}}}^{k} d \tilde{x}^{k}\right)^{t}\left(t^{k}+{\tilde{\alpha_{d}}}^{k} d \tilde{t}^{k}\right)
\end{aligned}
$$

Calcule $\sigma^{k}$ e $\mu^{k}$.

Calcule os resíduos $r_{5}^{k}$ e $r_{6}^{k}$.

$$
\begin{aligned}
r_{s p}^{k} & =r_{4}^{k}-\left(P^{k}\right)^{-1} r_{5}{ }^{k}+\left(S_{p}^{k}\right)^{-1} r_{6}{ }^{k}-\left(S_{p}^{k}\right)^{-1} W_{p}^{k} r_{2}^{k} \\
r_{a}^{k} & =r_{1}^{k}+D^{k} r_{s p}^{k} \\
r^{k} & =r_{a}^{k}+B \tilde{B}^{-1} r_{3}^{k} \\
v^{k} & =\left(B^{-t} \tilde{B} B^{-1}\right) r^{k} \\
d y^{k} & =v^{k}-\left(B^{-t} \tilde{B} B^{-1}\right) E\left(D^{-1}+Z\right)^{-1} E^{t} v^{k} \\
d \theta^{k} & =\tilde{B}^{-1}\left(B^{t} d y^{k}-r_{3}^{k}\right) \\
d p^{k} & =-\left(D_{p}^{k}\right)^{-1}\left(r_{p}^{k}+d y(p)^{k}\right) \\
d s_{p}^{k} & =r_{2}^{k}-d p^{k} \\
d z_{p}^{k} & =\left(P^{k}\right)^{-1}\left(r_{5}^{k}-Z_{p}^{k} d p^{k}\right) \\
d w_{p}^{k} & =\left(S_{p}^{k}\right)^{-1}\left(r_{6}^{k}-W_{p}^{k} d s_{p}^{k}\right) \\
d x^{k} & =\left(d p^{k}, d s_{p}^{k}\right) \\
d t^{k} & =\left(d z_{p}^{k}, d w_{p}^{k}\right) \\
\rho_{p}{ }^{k} & =\min _{d x_{i}{ }^{k}<0}\left\{-\frac{x_{i}{ }^{k}}{d x_{i}{ }^{k}}\right\} ; \quad \rho_{d}{ }^{k}=\min _{d t_{i}{ }^{k}<0}\left\{-\frac{t_{i}{ }^{k}}{d t_{i}{ }^{k}}\right\} \\
\alpha_{p}^{k} & =\min ^{k}\left(1, \tau \rho_{p}^{k}\right) ; \quad \alpha_{d}^{k}=\min \left(1, \tau \rho_{d}^{k}\right) ; \quad \alpha^{k}=\min \left(\alpha_{p}, \alpha_{d}\right) \\
\theta^{k+1} & =\theta^{k}+\alpha^{k} d \theta^{k} \\
x^{k+1} & =x^{k}+\alpha^{k} d x^{k} \\
y^{k+1} & =y^{k}+\alpha^{k} d y^{k} \\
t^{k+1} & =t^{k}+\alpha^{k} d t^{k}
\end{aligned}
$$

Até convergir.

Podemos destacar que a resolução dos sistemas lineares envolve a maior parte do esforço computacional do método. Então, utilizamos a fórmula de ShermanMorrison-Woodbury para resolver os sistemas lineares resultantes da eliminação de variáveis e, com isso, conseguimos reduzir o esforço computacional do método. Visto que as matrizes $B$ e $\tilde{B}$ não variam a cada iteração, apenas uma decomposição destas matrizes é necessária, e pode ser calculada antes do processo iterativo. Podemos verificar também que $\left(D^{-1}+Z\right)^{-1}$ pode ser calculada com pouco esforço computacional, uma vez que sua dimensão é dada pelo número de geradores, que geralmente é bem menor que o número de barras. 


\subsection{Inicialização, Parâmetros e Critério de Convergência}

Para a implementação do método de pontos interiores utilizaremos:

- $\tau=0.99995 ; \sigma=\frac{1}{\sqrt{n}}$.

O ponto inicial adotado foi:

- $\theta^{0}=0 ; p^{0}=s_{p}{ }^{0}=\frac{p^{\max }}{2} ; y^{0}=0$;

- $z_{p}{ }^{0}=w_{p}^{0}=e$.

O critério de convergência é dado por:

$$
\max \left\{\begin{array}{c}
\frac{\|l-B \theta+p\|}{1+\|l\|} \\
\frac{\left\|p^{m a x}-p-s_{p}\right\|}{1+\left\|p^{m a x}\right\|} \\
\frac{\left\|c_{p}+y(p)-z_{p}+w_{p}+H p\right\|}{1+\left\|c_{p}\right\|} \\
\frac{p^{t} z_{p}+s_{p} w_{p}}{1+\left\|\theta^{t} \tilde{B} \theta+p^{t} H p+c_{p}^{t} p+l^{t} y-p^{\text {maxt }} w_{p}\right\|}
\end{array}\right\} \leq \varepsilon .
$$

\section{Resultados Numéricos}

A implementação dos métodos de pontos interiores foi feita em MATLAB 5.3, e utilizamos os sistemas teste IEEE 30 e IEEE 118, os sistemas SSC 810, SSC 1654 e SSC 1732, que são três representações do sistema Sul - Sudeste - Centro-Oeste, e o sistema interconectado brasileiro, que possui 1993 barras.

Nestes experimentos, foi adotado o valor $p^{\text {min }}=0$ para os geradores. Para simplificar a interpretação dos resultados, somente funções quadráticas puras foram utilizadas, ou seja, $c_{p}=0$, e escolhemos o valor de tolerância $\varepsilon=10^{-5}$.

O tempo computacional em segundos, o número de iterações e de flops (número de operações de ponto flutuante) para cada um dos sistemas encontram-se nas Tabelas 1 e 2 para o método preditor-corretor para a modelagem por fluxos em rede $(\mathrm{MFR})$ e para a modelagem baseada no princípio do mínimo esforço (PME) do problema de fluxo de potência ótimo, respectivamente.

Tabela 1: Modelo de Fluxos em Rede.

\begin{tabular}{|l|r|r|r|}
\hline Sistema & iterações & tempo & flops \\
\hline IEEE 30 & 6 & 0,02 & 69704 \\
IEEE 118 & 7 & 0,15 & 470685 \\
SSC 810 & 4 & 2,85 & 3773277 \\
SSC 1654 & 5 & 10,80 & 9531362 \\
SSC 1732 & 5 & 11,67 & 9844461 \\
BRASIL & 4 & 10,29 & 5782112 \\
\hline
\end{tabular}


Tabela 2: Princípio do Mínimo Esforço.

\begin{tabular}{|l|r|r|r|}
\hline Sistema & iterações & tempo & flops \\
\hline IEEE 30 & 6 & 0,01 & 68468 \\
IEEE 118 & 4 & 0,09 & 541101 \\
SSC 810 & 3 & 2,63 & 3301261 \\
SSC 1654 & 2 & 41,35 & 16303972 \\
SSC 1732 & 2 & 48,71 & 18304309 \\
BRASIL & 2 & 28,74 & 10686731 \\
\hline
\end{tabular}

\title{
6. Conclusões
}

Observando os resultados das tabelas, podemos concluir que:

- Os sistemas de menor dimensão são resolvidos em um número maior de iterações que os sistemas maiores. Uma possível explicação para isso é que sistemas menores estão mais carregados.

- Os métodos de pontos interiores se mostraram robustos, convergindo rapidamente mesmo para problemas bastante sobrecarregados, sem apresentar instabilidade numérica. Podemos verificar que todas as abordagens são rápidas mesmo para sistemas muito carregados.

- Concluimos também que a modelagem baseada no princípio do mínimo esforço é superior à por fluxo em redes em número de iterações. Mas, para problemas de maior dimensão, a modelagem por fluxo em redes se mostra mais rápida, mesmo realizando mais iterações.

\begin{abstract}
In this work, the network flow formulation for the optimal DC power flow problem and the least effort principle formulation are presented. A specific predictor-corrector interior point method is developed for both power flow models. Numerical results in MATLAB confronting both approaches for large scale real systems are presented and the interior point method shows to be robust, achieving fast convergence in all instances tested.
\end{abstract}

\section{Agradecimentos}

Este trabalho contou com o apoio financeiro da Fundação de Amparo à Pesquisa do Estado de São Paulo (FAPESP) e Conselho Nacional de Desenvolvimento Científico e Tecnológico (CNPq).

\section{Referências}

[1] M.F. Carvalho, S. Soares e T. Ohishi, Optimal active power dispatch by network flow approach, IEEE Transactions on Power Systems, 3, No. 3 (1988), $1640-1647$. 
[2] G.H. Golub e C.F. Van Loan, "Matrix Computations", Third Edition, The Johns Hopkins University Press, Baltimore, Maryland, 1996.

[3] J.M. Martinez e L.T. Santos, Some New Theoretical Results on Recursive Quadratic Programming Algorithms, Journal of Optimization Theory and Applications, 97 (1998), 435-454.

[4] S. Mehrotra, On the implementation of a primal-dual interior point method, SIAM Journal on Optimization, 2, No. 4 (1992), 575-601.

[5] J.A. Momoh, M.E. El-Hawary e R. Adapa, A Review of Selected Optimal Power Flow Literature to 1993, Part II Newton, Linear Programming and Interior Point Methods, IEEE Transactions on Power Systems, 14, No. 1 (1999), 105111.

[6] R.D.C. Monteiro, I. Adler e M.G.C. Resende, A polynomial-time primal-dual affine scaling algorithm for linear and convex quadratic programming and its power series extension, Mathematics of Operations Research, 15 (1990), 191214.

[7] A. Monticelli, A. Santos, M.V. Pereira, S.H. Cunha, B.J. Parker e J.C. Praça, Interactive transmission network planning using a least effort criterion, IEEE Transactions on Power Apparatus and Systems, 10 (1982), 3919-3925.

[8] A.R.L. Oliveira e A.M. Lima, Métodos de pontos interiores aplicado ao fluxo de potência ótimo usando o princípio do mínimo esforço - Comparação com o modelo de fluxos em rede, em "Anais da XXXV Conferência da SOBRAPO", ISSN 1518-1731, Natal, Brasil, pp. 1993-2004, Sociedade Brasileira de Pesquisa Operacional, novembro, 2003.

[9] A.R.L. Oliveira, L. Nepomuceno e S. Soares, Optimal active power dispatch combining network flow and interior point approaches, IEEE Transactions on Power Systems, 18 (2003), 278-285.

[10] A.R.L. Oliveira e S. Soares, Métodos de Pontos Interiores para Problema de Fluxo de Potência Ótimo DC, SBA: Controle $\mathscr{E}$ Automação, 14, No. 3 (2003), $278-285$.

[11] V.H. Quintana, G.L. Torres e J. Medina-Palomo, Interior point methods and their applications to power systems: A classification of publications and software codes, IEEE Transactions on Power Systems, 15, No. 1 (2000), 170-176.

[12] D.F. Shanno e R.J. Vanderbei, Interior-point methods for nonconvex nonlinear programming: Orderings and higher-order methods, Mathematical Programming, 87 (2000), 303-316. 
